\title{
Quality of Life of Patients Using the Hemodialysis Reliable Outflow (HeRO) Graft in Hemodialysis
}

\author{
Muhammad Haisum Maqsood $^{1}$, Kinza Rubab ${ }^{2}$ \\ 1. Epidemiology and Public Health, King Edward Medical University / Mayo Hospital, Lahore, PAK 2. Internal Medicine, \\ King Edward Medical University / Mayo Hospital, Lahore, PAK
}

Corresponding author: Muhammad Haisum Maqsood, haisumbajwa@live.com

\begin{abstract}
End-stage renal disease (ESRD) is one of the most feared consequences of kidney disease. A large number of patients with ESRD require long-term hemodialysis. Vascular access options for hemodialysis include the placement of arteriovenous (AV) fistulas, AV grafts, and tunneled dialysis catheters (TDCs). An alternative to the TDC is the Hemodialysis Reliable Outflow (HeRO; Cryolife Inc., Eden Prairie, MN, USA) Graft. The HeRO Graft has been designed to overcome the development of central venous stenosis or occlusion. The objective is to evaluate the quality of life of patients using the HeRO Graft in end-stage renal disease for hemodialysis. We searched PubMed, Medical Literature Analysis and Retrieval System Online (MEDLINE), Excerpta Medica dataBASE (EMBASE), Cumulative Index to Nursing and Allied Health Literature (CINHAL), Directory of Open Access Journals (DOAJ), Pubpsych, and Google Scholar on October 30, 2018. We included published articles in the English language that used the HeRO Graft for ESRD. The adequacy of dialysis and bacteremia rates proved to be equal to those of conventional $\mathrm{AV}$ grafts. It turned out that 2.21 interventions per year were needed to maintain the patency of the HeRO Graft while only 1.17 interventions were needed to maintain the patency of the lower extremity graft. Mortality, ischemia, and infection rates were similar for both groups. The tunneled dialysis catheters have a higher incidence of infection as compared to the HeRO Graft. The initial device and placement costs for the HeRO Graft were higher than those for TDCs but savings from the lower incidence of device complications and longer effective device patency make it cost-effective. Based on the limited evidence, it has been discerned that the HeRO Graft is an optimal option for hemodialysis in patients of ESRD who have exhausted all means of upper extremity access. Though almost similar to the AV grafts in terms of complications and less functional than femoral grafts, it still outclasses them in improving the quality of life of such patients.
\end{abstract}

Received 01/04/2019 Review began 01/07/2019 Review ended 01/17/2019 Published 01/19/2019

๑) Copyright 2019 Maqsood et al. This is an open access article distributed under the terms of the Creative Commons Attribution License CC-BY 3.0., which permits unrestricted use, distribution, and reproduction in any medium, provided the original author and source are credited.
Categories: Internal Medicine, Nephrology, Healthcare Technology

Keywords: hero graft, hemodialysis, end stage renal disease

\section{Introduction And Background}

\section{The condition}

End-stage renal disease (ESRD) is one of the most feared consequences of kidney disease. It affects over 500,000 patients in the United States and is increasing in prevalence, with over 100,000 new cases reported each year [1]. ESRD occurs when kidney function has deteriorated to an extent that it is no longer adequate to sustain life, unless renal replacement therapy, dialysis, or transplantation is done [2].

A large number of patients with ESRD require long-term hemodialysis (HD), which is a life-preserving therapy [3]. According to estimates, more than 1.5-million patients receive regular HD treatment worldwide, with the number growing at an annual rate of around $7 \%$ [4]. With the increased life expectancy of dialysis patients, the challenges of maintaining dialysis access increase as well [5].

\section{The intervention}

Vascular access options for hemodialysis include the placement of arteriovenous (AV) fistulas, AV grafts, and tunneled dialysis catheters (TDCs) [6]. An alternative to the TDC is the Hemodialysis Reliable Outflow (HeRO; Cryolife Inc., Eden Prairie, MN, USA), which was approved by the United States Food and Drug Administration (USFDA) as a graft for use in ESRD in 2008 [6]. Patients with end-stage renal disease who require hemodialysis are at risk for the development of central venous stenosis or occlusion, with an estimated prevalence of $16 \%$ to $50 \%$ [7-9]. The HeRO Graft has been designed to overcome these limitations, but it typically requires several weeks for tissue incorporation [10].

\section{How the intervention works}

The HeRO Graft is entirely subcutaneous. It consists of two primary components: a conventionally expanded polytetrafluoroethylene (ePTFE) graft component and a silicone venous outflow component. The ePTFE graft component is placed in the upper arm and anastomosed to the target artery for arterial inflow, and the venous outflow component is placed similarly to a TDC with its distal end terminating at the cavoatrial 


\section{Cureus}

junction. Two components are placed entirely subcutaneously. When they are brought together via a titanium connector, it results in the shunting of arterial blood from the donor artery into the central venous system, thereby bypassing the need for creating a formal venous anastomosis for outflow [11-13]. Hence, for patients with no adequate upper extremity peripheral venous outflow, the HeRO Graft can provide an opportunity for a preferred upper extremity subcutaneous AV dialysis graft.

\section{The importance of this review}

The absence of reviews that have assessed the quality of life of patients using the HeRO Graft has prompted us to evaluate the available evidence to establish the benefits and harms of the HeRO Graft in relation to different hemodialysis techniques in patients with ESRD.

\section{Objective}

To evaluate the quality of life of patients using the HeRO Graft in end-stage renal disease for hemodialysis.

\section{Review}

Methods

Search Methods for the Identification of Studies

A systematic review of the current published literature on the Hemodialysis Reliable Flow (HeRO) Graft for hemodialysis in ESRD, in accordance with Preferred Reporting Items for Systematic Reviews and Metaanalyses (PRISMA), was undertaken. A systemic search for systematic reviews, meta-analyses, multicenter studies, and randomized controlled trials was made using the search terms "HeRO Graft," "Hemodialysis," and "End Stage Renal Disease." We systematically searched the PubMed, Medical Literature Analysis and Retrieval System Online (MEDLINE), Excerpta Medica dataBASE (EMBASE), Cumulative Index to Nursing and Allied Health Literature (CINHAL), Directory of Open Access Journals (DOAJ), Pubpsych, and Google Scholar on October 30, 2018. Nine articles were selected and included in this study. Since most of the existing articles dated back to at least 10 years ago, there was no need to set a time limit. The inclusion and exclusion criteria have been listed in Table 1 . The exclusion criteria include unpublished articles, conference articles, commentaries, letters to the editor, and reports in a language other than English. We also excluded those articles that did not mention quality of life issues.

\begin{tabular}{|l|l|}
\hline Inclusion Criteria & Exclusion Criteria \\
\hline Published articles & Unpublished articles \\
\hline Article text in English & Language other than English \\
Articles related to the HeRO Graft & Unrelated articles \\
ESRD patients & Irrelevant patients \\
\hline
\end{tabular}

\section{TABLE 1: Inclusion and exclusion criteria}

HeRO: Hemodialysis Reliable Outflow (Cryolife Inc; Eden Prairie, MN, USA); ESRD: End-stage renal disease

Data Extraction and Analysis

No statistical analyses or meta-analyses were conducted. Instead, the existing results and conclusions presented in the reviews were extracted and reported in a systematic format.

Results

The initial search produced 806 results; 37 qualified for the full-text review. Ultimately, nine were selected, as shown in Figure 1. A quality assessment of the studies was performed per the Grading of Recommendations Assessments, Development, and Evaluation (GRADE) guidelines. 


\section{Cureus}

806 Potentially Relevant Citations Identified and Screened for

Retrieval:

4 articles from Directory of Open Access Journals.

500 articles from Google scholar.

188 articles from ProfSearch.

52 articles from PubMed.

30 articles from MEDLINE.

27 articles from Embase.

5 articles from Pubpsych.

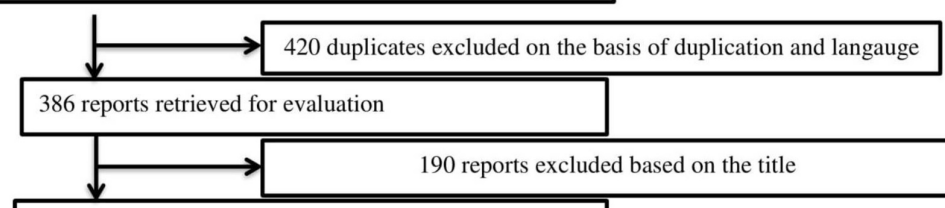

196 reports retrieved for evaluation

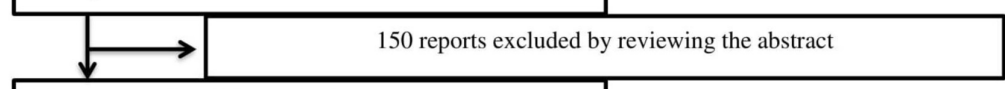

46 reports retrieved for evaluation

9 reports retrieved for final evaluation

FIGURE 1: Flow diagram of literature search results

\section{Descriptive analyses/findings}

Comparing It to the AV Graft

According to a study included in this review, which involved data collection from 72 subjects ( 52 HeRO Grafts and $20 \mathrm{AV}$ grafts), primary and secondary patency rates were $34.8 \%$ and $67.6 \%$ for the HeRO Graft and30.6\% and $58.4 \%$ in the control group, thus being almost similar. The intervention rates were $2.2 /$ year for the HeRO Graft and 1.6/year for the control $(\mathrm{p}=0.100)$. The adequacy of dialysis and bacteremia rates proved to be equal to those of conventional AV grafts [14].

Comparison with the Femoral Graft

According to a study included in this review, it turned out that 2.21 interventions per year were needed to maintain patency for the HeRO Graft while only 1.17 interventions were needed to maintain the patency of the lower extremity graft. Mortality, ischemia, and infection rates were similar for both groups. The lower extremity graft had an infection rate of 0.71 per 1000 days and the HeRO had 0.61 per 1000 days. Secondary patency at 12 months for the HeRO Graft was $42 \%$ and the thigh graft was $86 \%$ [6].

Another study from our review, which had been conducted with 35 femoral AV (fAVG) grafts and 21 HeRO placements showed a primary patency of $40.5 \%, 18.7 \%$, and $14.9 \%$ for fAVG and $29.0 \%, 29.0 \%$, and $0 \%$ for HeRO at six months, 12 months, and two years $(\mathrm{p}=0.67)$, respectively. Assisted primary patency was also similar, with $43.8 \%, 29.4 \%$, and $13.8 \%$ for fAVG and $34.8 \%, 34.8 \%$, and $17.4 \%$ for HeRO at six months, 12 months, and two years $(\mathrm{p}=0.81)$, respectively. Secondary patency was $62.6 \%, 50.6 \%$, and $19.3 \%$ for fAVG and $68.0 \%, 53.5 \%$, and $38.3 \%$ for HeRO at six months, 12 months, and two years ( $\mathrm{p}=0.69)$, respectively. The average number of interventions to maintain patency for fAVG was $1.1 \pm 1.47$ and $1.65 \pm 2.52$ for HeRO $(\mathrm{p}=0.35)$. Infectious rates were $29 \%$ in fAVG and $15 \%$ in HeRO ( $\mathrm{p}=0.33)$ [2].

Does It Outclass the Tunneled Catheters?

The results have shown that the tunneled dialysis catheters have a higher incidence of infection as compared to the HeRO Graft and fAVG, suggesting that their use is preferable to catheter dependence [15]. 
It has been proved in one of the studies included in our reviews that TDCs are the least desirable due to less effective dialysis, an increased risk of thrombosis, higher rates of bacteremia, and greater mortality [16-17].

Infections

Early infection was defined as episodes of bacteremia or HeRO infections requiring resection within 30 days of HeRO implantation.

The rate of HeRO Grafts being resected due to infectious complications was 0.41 in directly placed grafts and 0.12 in staged grafts per 1000 implant days [13].

The study of Gage et al. [14] has reported an access-related infection incidence of 0.14/1000 implant days in patients with HeRO, and Katzman et al. [12] have reported a HeRO-related infection incidence of 0.71/1000 implant days.

The Two-staged Technique

In the two-staged technique, the overall rate of infection was $8.6 \%$ for primary HeRO implantations and $2.3 \%$ for staged implantations $(\mathrm{p}=.12)$. The rates of early bacteremia and HeRO resection requiring surgical resection were not significantly different between the groups ( $\mathrm{p}=.19$ and $\mathrm{p}=.065$, respectively) [13].

Maintaining the "Real Estate"

Glickman's study concluded that in patients likely to be on dialysis for more than three to five years, the HeRO device should be considered, as it provides patency rates similar to upper extremity grafts and maintains lower extremity "real estate" [18].

Cost-effectiveness

The study in our review (conducted on 100 patients with HeRO Grafts) reported six fewer failed devices, 53 fewer access-related infections, and 67 fewer device thromboses as compared to patients managed with tunneled catheters. The initial device and placement costs for the HeRO Graft were higher than those for TDCs but savings from the lower incidence of device complications and longer effective device patency make it cost-effective [3].

\section{Modified Hero Graft}

The HeRO Graft has a drawback: it requires several weeks for tissue incorporation. The ACUSEAL graft (Gore Technologies, Newark, DE, US), a modified HeRO Graft, allows immediate cannulation, thus reducing catheter dependence time and its associated complications. Out of the 10 modified HeRO Grafts placed, postoperative complications included two thromboses and one hematoma. The primary and secondary patency rates were $70 \%$ and $90 \%$, respectively [11].

\section{Discussion}

This systematic review aimed to assess the quality of life of the patients of ESRD using the HeRO Graft for hemodialysis. It included nine studies and has revealed the significant pros and cons of this innovative technique. Among the existing vascular access options, such as conventional AV fistulas, AV grafts, femoral grafts, and tunneled dialysis catheters, the HeRO Graft is a novel addition.

The results of this systematic review signify that the HeRO Graft has patency, rate of infection, intervention, and mortality almost equal to that of a conventional AV graft. But the increased risk of central vein stenosis and occlusion has necessitated the use of an alternative technique such as the HeRO Graft or femoral AV grafts. Though the femoral grafts have higher patency and a low rate of infection and intervention, HeRO Grafts are a better option, as they help preserve the lower extremities for any future access.

Since the HeRO Graft requires time for tissue incorporation in order to get functional, the tunneled dialysis catheters have to be used as an interim. This problem has been addressed with the modified HeRO Graft that requires early cannulation; thus reducing the rate of infection, thrombosis, and the inadequacy of dialysis associated with catheter dependence. The systematic review of ours also suggested that though the initial cost of the HeRO Graft is higher than that of other hemodialysis techniques, the low rate of complications makes it cost-effective.

While valuable information has been gained from this review, a significant issue was the marked heterogeneity among studies about the aspect of the HeRO Graft they tended to focus on. Our review indicates that the results of most of the studies incline toward the HeRO Graft being the optimal option for those patients of ESRD who have exhausted all means of upper extremity access. 
The results of our systematic review endorse the already existing renowned studies of Wallace, Gage, Nassar, and Katzman. Our review also revealed a significant paucity of evidence regarding the impacts of the HeRO Graft on quality of life. The existing studies have not systematically evaluated the HeRO Graft, an emerging mode of hemodialysis. Another drawback of the available literature is that it fails to systematically evaluate the complications associated with the HeRO implants. The limitations of the included studies did not enable us to derive conclusive evidence about the adverse outcomes if any.

A limitation of our review is the small number of studies for the systematic analysis and the possibility that relevant studies may have not been included. Some clinically significant parameters have also not been addressed, which is another drawback. The low methodological quality meant that robust conclusions could not be derived. The included studies did not provide sufficient information to assess if they were free of selective reporting. Moreover, we could only find a few parameters to assess quality of life such as infectious complications and vascular access. Despite these limitations, our systematic review holds an important place, as it has been one of the few studies to address the quality of life of patients using the HeRO Graft.

Currently, there is insufficient evidence to establish facts. Suitable clinical research needs to be done to establish the superiority of one hemodialysis technique over the existing ones and to know whether the HeRO Graft is an effective and optimal mode of hemodialysis or not.

\section{Conclusions}

Many of the studies evaluating the HeRO Graft as a mode of dialysis are small, outdated, of poor quality, and have insufficient evidence to establish the role of this mode of hemodialysis. However, it can be concluded that the HeRO Graft excels over the tunneled dialysis catheters but is almost equal to the AV grafts in terms of complications and reinterventions. Though the HeRO Graft is less effective than femoral grafts, it is still an optimal option, as it preserves access to the lower extremities.

\section{Additional Information \\ Disclosures}

Conflicts of interest: In compliance with the ICMJE uniform disclosure form, all authors declare the following: Payment/services info: All authors have declared that no financial support was received from any organization for the submitted work. Financial relationships: All authors have declared that they have no financial relationships at present or within the previous three years with any organizations that might have an interest in the submitted work. Other relationships: All authors have declared that there are no other relationships or activities that could appear to have influenced the submitted work.

\section{References}

1. Rocco MV, Berns JS: KDOQI clinical practice guideline for diabetes and CKD: 2012 update . Am J Kidney Dis. 2012, 60:850-886. 10.1053/j.ajkd.2012.07.005

2. Kudlaty EA, Pan J, Allemang MT, Kendrick DE, Kashyap VS, Wong VL: The end stage of dialysis access: femoral graft or HeRO vascular access device. Ann Vasc Surg. 2015, 29:90-97. 10.1016/j.avsg.2014.06.001

3. Al Shakarchi J, Inston N, Jones RG, Maclaine G, Hollinworth D: Cost analysis of the Hemodialysis Reliable Outflow (HeRO) Graft compared to the tunneled dialysis catheter. J Vasc Surg. 2016, 63:1026-1033. 10.1016/j.jvs.2015.10.089

4. Grassmann A, Gioberge S, Moeller S, Brown G: ESRD patients in 2004: global overview of patient numbers, treatment modalities and associated trends. Nephrol Dial Transplant. 2005, 4:2587-2593. 10.1093/ndt/gfi159

5. The National Kidney and Urologic Diseases Information Clearinghouse (NKUDIC). Kidney disease statistics for the United States. (2012). Accessed: January 24, 2013: https://www.niddk.nih.gov/healthinformation/kidney-disease.

6. Schild AF: Maintaining vascular access: the management of hemodialysis arteriovenous grafts . J Vasc Access. 2010, 11:92-99. 10.1177/112972981001100202

7. Glickman MH: HeRO graft versus lower extremity grafts in hemodialysis patients with long standing renal failure. J Vasc Access. 2016, 17:30-31. 10.5301/jva.5000531

8. Agarwal AK: Central vein stenosis: current concepts. Adv Chronic Kidney Dis. 2009, 16:360-370. 10.1053/j.ackd.2009.06.003

9. MacRae JM, Ahmed A, Johnson N, Levin A, Kiaii M: Central vein stenosis: a common problem in patients on hemodialysis. ASAIO Journal. 2005, 51:77-81. 10.1097/01.MAT.0000151921.95165.1E

10. Yevzlin AS: Hemodialysis catheter-associated central venous stenosis [Epub] . Semin Dial. 2008, 21:522-527. 10.1111/j.1525-139X.2008.00496.x

11. Perry J, Hardy D, Agarwal S, Agarwal G: Safety and efficacy of a modified HeRO dialysis device in achieving early graft cannulation: A single-institution experience. J Vasc Surg Cases Innov Tech. 2017, 3:175-179.

12. Glickman MH: HeRO vascular access device. Semin Vasc Surg. 2011, 24:108-112. 10.1053/j.semvascsurg.2011.05.006

13. Katzman HE, McLafferty RB, John RR, Glickman MH, Peden EK, Lawson JH: Initial experience and outcome of a new hemodialysis access device for catheter-dependent patients. J Vasc Surg. 2009, 50:600-607. 10.1016/j.jvs.2009.04.014

14. Gage SM, Katzman HE, Ross JR, Hohmannd SE, Sharpe CA, Butterly DW, Lawson JH: Multi-center experience of 164 consecutive Hemodialysis Reliable Outflow [HeRO] graft implants for hemodialysis treatment. Eur J 


\section{Cureus}

Vasc Endovasc Surg. 2012, 44:93-99. 10.1016/j.ejvs.2012.04.011

15. Nassar GM, Glickman MH, McLafferty RB, et al.: A comparison between the HeRO Graft and conventional arteriovenous grafts in hemodialysis patients. Semin Dial. 2014, 27:310-318. 10.1111/sdi.12173

16. Shingarev R, Barker-Finkel J, Allon M: Natural history of tunneled dialysis catheters placed for hemodialysis initiation. J Vasc Interv Radiol. 2013, 24:1289-1294. 10.1016/j.jvir.2013.05.034

17. Ravani P, Gillespie BW, Quinn RR, et al.: Temporal risk profile for infectious and noninfectious complications of hemodialysis access. J Am Soc Nephrol. 2013, 24:1668-1677. 10.1681/ASN.2012121234

18. Ladenheim E, Lulic D, Lum C, Agarwal S: Primary and secondary patencies of transposed femoral vein fistulas are significantly greater than with the HeRO graft. J Vasc Access. 2017, 18:232-237. 10.5301/jva.5000697 